\title{
Development and External Validation a Novel Inflammation-Based Score for Acute Kidney Injury and Prognosis in Intensive Care Unit Patients
}

\author{
Jingjing Wan ${ }^{1, *}$ \\ Gaorui Zou ${ }^{2, *}$ \\ Bo $\mathrm{He}^{1}$ \\ Chao Zhang ${ }^{3}$ \\ Yanfang Zhu' \\ Lan Yin $^{3}$ \\ Zhibing Lu'
}

'Department of Cardiology, Zhongnan Hospital of Wuhan University, Wuhan,

43007I, People's Republic of China;

${ }^{2}$ Department of Anesthesiology, Wuhan

No. I Hospital, Wuhan, 430022, People's

Republic of China; ${ }^{3}$ Department of

Cardiology Electrocardiogram, Zhongnan

Hospital of Wuhan University, Wuhan,

43007I, People's Republic of China

*These authors contributed equally to this work

\begin{abstract}
Purpose: We aimed to evaluate the predictive ability of an integrated score based on several inflammatory indices of acute kidney injury (AKI) in patients in the intensive care unit (ICU).

Patients and Methods: In this observational study, 2555 patients from the Medical Information Mart for Intensive Care III database were randomly assigned to the test set $(n=1599)$ and internal validation set $(n=656)$. Moreover, 412 coronary care unit patients from Zhongnan Hospital, Wuhan University were also included in the external validation set. The AKI-specific inflammatory index (ASII) was created using various inflammatory indices significantly associated with AKI. We further developed and validated two nomograms based on the ASII and other informative clinical features of AKI and prognosis.

Results: The ASII was calculated as $2.317 \times \mathrm{MLR}+0.417 \times \mathrm{GPS}+0.007 \times \mathrm{ALRI}$. In the training set, patients with a high ASII had a higher risk of incident AKI (odds ratio [OR], 5.33; 95\% confidence index $[\mathrm{CI}], 3.60-7.88 ; \mathrm{P}<0.001)$ than those with a low ASII with or without preexisting chronic kidney disease. The nomograms for AKI and prognosis based on the ASII and other significant clinical characteristics had high predictive value in the prediction of AKI and prognosis in patients in the ICU. Moreover, the results in the internal validation set and in the external validation cohort were almost consistent with those in the training set.

Conclusion: The ASII is an AKI-specific tool based on the combination of available inflammatory indices. A high ASII is a strong predictor of a higher risk of AKI and worse survival outcomes in patients in the ICU.
\end{abstract}

Keywords: acute kidney injury, inflammation, AKI-specific inflammatory index, intensive care unit, nomogram

\section{Introduction}

Acute kidney injury (AKI), which refers to a group of syndromes leading to a sharp decrease in glomerular filtration, is one of the most life-threatening complications in hospitalized patients worldwide. ${ }^{1-3}$ As AKI can lead to the development of chronic kidney disease (CKD) or end-stage renal disease, the effect of AKI on longterm health and cost may be far greater than that previously estimated. ${ }^{2,3}$ Thus, screening for patients who are at high risk of AKI may result in earlier diagnosis, avoidance of potentially nephrotoxic exposures, lower health care expenses, and better long-term prognosis. ${ }^{4,5}$

Inflammation is a complex biological response that is mainly generated by innate immune cells. AKI is characterized by intrarenal and systemic inflammation.
Correspondence: Zhibing Lu; Lan Yin Department of Cardiology, Zhongnan Hospital of Wuhan University, No. 169

Donghu Road, Wuhan, 43007I, People's Republic of China

Tel +86 27-678/2999

Email zhibinglu123@I26.com;

779367161@qq.com 
A systematic inflammatory response can be detected using a variety of hematological markers, such as the neutrophil-tolymphocyte ratio (NLR), ${ }^{6,7}$ monocyte-to-lymphocyte ratio (MLR), platelet-to-lymphocyte ratio (PLR), ${ }^{8,9}$ and C-reactive protein-to-albumin ratio (CAR), ${ }^{10}$ some of which have been reported to be closely associated with the incidence of AKI in different populations. As the multidirectional relationship between AKI, inflammation, and immunity depends on an intricate network, the clinical benefit of individual biomarkers may be useless in clinical practice due to their low predictive efficiencies. Therefore, a combination of different immuneinflammatory indices may better reflect inflammatory status and improve predictive performance than individual biomarkers. However, thus far, no combined inflammatory score has been specifically designed for risk stratification in patients in intensive care units (ICUs). Hence, in this two-stage observational study, we initially developed a novel AKI-specific inflammatory index (ASII) for AKI in patients in the ICU using data from the Medical Information Mart for Intensive Care (MIMIC) III database. We further combined the novel index with other common clinical variables to create and verify nomograms for the prediction of AKI and mortality in patients in the ICU. Finally, we validated these results in patients in the coronary care unit (CCU) of our hospital.

\section{Materials and Methods}

\section{Study Population}

We first obtained data from the MIMIC III database. ${ }^{11}$ A freely available to the medical research community, which consists of more than 46,000 ICU patients admitted to Beth Israel Deaconess Medical Center (Boston, MA, USA) from 2001 to 2012. Access to the database for research was approved by the Institutional Review Boards of the Massachusetts Institute of Technology (Cambridge, MA, USA) and the Beth Israel Deaconess Medical Center and was granted a waiver of informed consent. We selected all adults in the database without missing results of white blood count, platelet, neutrophil, lymphocyte, monocyte, C-reactive protein and albumin. Since there were not a clear age of patients with 89 or older, a median age of 91.4 years old was taken the place of them. A total of 2555 patients were included in the study. All patients were randomly assigned to the training and internal validation sets at a ratio of 7:3 (training cohort, $n=1599$; internal validation cohort, $\mathrm{n}=656$ ). We prospectively included 412 patients in the training cohort who were admitted to the $\mathrm{CCU}$ at Zhongnan Hospital of Wuhan University from January 1, 2014 to June 1, 2015 , as previously reported. ${ }^{12}$

\section{Inflammatory Indexes}

All initial biochemical data on admission were collected from the MIMIC III database for the patients enrolled in this study. We calculated the patients' baseline characteristics using the SQL code available in the MIMIC code repository. The following inflammatory indexes were evaluated: the NLR, PLR, MLR, systemic immune-inflammation index (SII), aspartate aminotransferase-to- lymphocyte ratio (ALRI), CAR, Glasgow prognostic score (GPS), modified GPS (mGPS), and prognostic index (PI). The NLR was defined as the ratio of neutrophils to lymphocytes. The PLR was defined as the ratio of platelets to lymphocytes. The MLR was calculated by dividing monocytes by lymphocytes. The SII was computed by the formula platelet count $x$ neutrophil count/lymphocyte count. The ALRI was defined as the ratio of aspartate aminotransferase to lymphocytes. The CAR was defined as the ratio of C-reactive protein and albumin. The cut-off values of the above inflammatory indices were identified using X-tile software. ${ }^{13}$ Moreover, the GPS, mGPS, and PI were defined and categorized according to previous studies. ${ }^{14,15}$

\section{Outcomes}

The primary outcome was the incidence of AKI, defined according to the KDIGO Clinical Practice Guidelines for AKI based on the serum creatinine criteria within 7 days after hospital admission. It was evaluated using the SQL code that was available in the MIMIC code repository. ${ }^{16}$ Moreover, we did not use urine output for AKI diagnosis because an indwelling urinary catheter may not be present in all patients. ${ }^{17}$ The estimated glomerular filtration rate (eGFR) was calculated using the CKD-EPI equation. ${ }^{18}$

The secondary outcomes included in-hospital mortality and 2-year mortality, defined as the period from admission to the end of the 2-year follow-up period. Mortality data of the discharged patients in the MIMIC III database was obtained from the US Social Security Death Index.

\section{Statistical Analyses}

X-tile version 3.6.1 and $\mathrm{R}$ software (version 3.6.1, http:// www.r-project.org) were used for all analyses. We used a two-sample $t$-test or the Mann-Whitney $U$-test to compare continuous variables between groups and Pearson's chisquare test to compare categorical variables between groups. To develop a novel inflammatory score specific to AKI, multivariable logistic regression was employed to select single inflammatory indices significantly associated with 
AKI. The training set was used to develop a novel inflammatory score, which was established based on the $\beta$ coefficients from multivariable logistic analysis. ASII scores were divided into three risk groups (low, intermediate, and high risk) using the 50th and 85th percentiles as cut-off values. Moreover, we combined ASII scores with other clinical features with $\mathrm{P}$ values of $<0.05$ in multivariable analysis to construct nomograms for AKI and prognosis. Discrimination as well as calibration were assessed using the $\mathrm{C}$-index and time-dependent receiver operating characteristic curves (td-ROC) as well as calibration curves, respectively. All statistical tests were two tailed, and a $\mathrm{P}$ value of $<0.05$ was considered statistically significant.

\section{Results}

\section{Patient Clinical Features}

A total of 2967 patients (1599 patients in the training cohort, 656 patients in the internal validation cohort, and 412 patients in the external validation cohort) were analyzed in this study. According to KDIGO-AKI criteria, 804 $(50.3 \%)$ patients in the training set, $312(47.6 \%)$ patients in the internal validation cohort, and $130(31.6 \%)$ patients in the external validation cohort had AKI during their ICU stay. The best cut-off values for inflammatory indicators, determined using X-tile software, are presented in Table 1.

We grouped the patients according to the presence and absence of AKI. In the training set, compared patients without AKI, those with AKI were older, had more comorbidities, had higher sequential organ failure assessment (SOFA) scores and total bilirubin levels, and lower mean arterial pressure (MAP), eGFR, and hemoglobin levels. Moreover, patients with AKI had higher values of inflammatory indicators, except the SII and CAR, than those without AKI. However, sex distribution, body mass index (BMI), and medication use, except diuretic use, were comparable between the groups. Moreover, patients in the internal validation and external validation cohorts exhibited similar characteristics to those in the test cohort (Table 1).

\section{Development of the Novel Inflammatory Index for AKI}

For developing the ASII, we only selected the informative inflammatory indexes that were significantly related to AKI in logistic regression analyses. We found that higher MLR, GPS, and ALRI values were independently associated with a higher risk of AKI (Table 2). Therefore, based on the $\beta$ - coefficient, the ASII for AKI was calculated as $2.317 \times$ MLR $+0.417 \times$ GPS $+0.007 \times$ ALRI. Using $50 \%(2.16)$ and $85 \%$ (4.15) centiles as cut-off values, ASII scores were classified into three different risk groups in the training and validation cohorts. As illustrated in Figure S1A-O, the ASII was significantly associated with AKI, CKD, eGFR, in-hospital mortality, and 2-year mortality in the training, internal validation set, and external validation sets.

We further confirmed the ASII score through multivariable logistic analysis, and after adjusting for other clinical factors, ASII seemed to be a robust marker for AKI with or without pre-existing CKD. Patients in the high-risk group had a five-fold higher risk of AKI than those in the low-risk group. These results were further verified in the internal validation and external validation cohorts (Table 3). After adjusting for age, sex, BMI, hypertension, diabetes, preexisting CKD, mean arterial pressure, hemoglobin level, SOFA score, serum albumin level, total bilirubin level, eGFR, treatment with loop diuretics, treatment with angiotensin-converting enzyme inhibitors/angiotensin receptor blocker (ACEI/ARB), and treatment with aspirin, the highrisk group had a higher risk of AKI than the low-risk group in the internal validation cohort (odds ratio, OR, 6.09, 95\% confidence index $[\mathrm{CI}], 3.32-11.16, \mathrm{P}<0.001)$ and the external validation cohort (OR, 3.09, 95\% CI, 1.04-5.31, $\mathrm{P}<0.001$ ). Regarding secondary outcomes, the ASII was a strong predictor of in-hospital mortality and 2-year mortality in the training, internal validation, and external validation cohorts (Figure 1A-F).

Moreover, for predicting AKI, the area under the receiver operating characteristic curve (AUC) was 0.786 in the training set, and a cut-off value of 1.93 yielded good specificity (80.3\%) and sensitivity (64.9\%).

\section{Development and Validation of a Nomogram for AKI}

Based on the results of multivariable logistic analysis, six variables were finally included in the nomogram for AKI - age, ASII, HGB, eGFR, SOFA score, and diabetes (Figure 2A). The C-index was used to evaluate the discrimination abilities of the nomogram, and the values were 0.821 (95\% CI, 0.800-0.841) in the training cohort, 0.797 (95\% CI, 0.763-0.831) in the internal validation set, and 0.895 (95\% CI, 0.864-0.927) in the external validation set, indicating that the nomogram had a good model discriminative capacity. The calibration curves for AKI are shown in Figure $3 \mathrm{~A}-\mathrm{C}$. The actual and the predicted probabilities of 
Table I Clinicopathological Characteristics of All Patients

\begin{tabular}{|c|c|c|c|c|c|c|c|c|c|}
\hline \multirow[t]{2}{*}{ Characteristics } & \multicolumn{3}{|c|}{ Training Set $(n=1599)$} & \multicolumn{3}{|c|}{ Internal Validation Set $(n=656)$} & \multicolumn{3}{|c|}{ External Validation Set $(n=4 \mid 2)$} \\
\hline & AKI (+) & AKI (-) & $P$ value & AKI (+) & AKI (-) & $P$ value & $\mathrm{AKI}(+)$ & AKI (-) & $P$ value \\
\hline $\mathrm{N}$ & 804 & 795 & - & 308 & 348 & - & 130 & 282 & \\
\hline Age (years) & $62.2 \pm 15.8$ & $60.3 \pm 17.2$ & 0.020 & $62.5 \pm 16.7$ & $61.9 \pm 21.4$ & 0.672 & $68.2 \pm 13.1$ & $65.5 \pm 13.1$ & $<0.001$ \\
\hline Sex, male, n (\%) & $445(55.3)$ & $447(56.2)$ & 0.724 & $185(60.1)$ & $188(54.0)$ & 0.119 & $42(32.3)$ & $93(33.0)$ & 0.893 \\
\hline BMI, $\mathrm{kg} / \mathrm{m}^{2}$ & $28.0 \pm 8.2$ & $27.9 \pm 8.1$ & 0.657 & $28.6 \pm 8.4$ & $28.1 \pm 7.4$ & 0.425 & $23.7 \pm 3.3$ & $23.8 \pm 3.5$ & 0.870 \\
\hline \multicolumn{10}{|l|}{ Primary diagnosis, n (\%) } \\
\hline ACS & $14(1.7)$ & $13(1.6)$ & 0.869 & $7(2.3)$ & $7(2.0)$ & 0.818 & $89(68.5)$ & $179(63.5)$ & 0.121 \\
\hline Acute heart failure & $113(14.1)$ & $54(6.8)$ & $<0.001$ & $34(11.0)$ & $17(4.9)$ & 0.003 & II (8.5) & $5(1.8)$ & 0.001 \\
\hline \multicolumn{10}{|l|}{ Preexisting diseases } \\
\hline CKD, n (\%) & $254(31.6)$ & $87(10.9)$ & $<0.001$ & III (36.0) & $29(8.3)$ & $<0.001$ & $42(32.3)$ & $17(6.0)$ & 0.001 \\
\hline Hypertension, n (\%) & $248(30.8)$ & $299(37.6)$ & 0.004 & $86(27.9)$ & $138(39.7)$ & 0.002 & $90(69.2)$ & $118(41.8)$ & $<0.001$ \\
\hline Diabetes, n (\%) & $315(39.2)$ & $174(21.9)$ & $<0.001$ & $116(37.7)$ & $98(28.2)$ & 0.010 & $63(48.5)$ & $79(28.0)$ & $<0.001$ \\
\hline \multicolumn{10}{|l|}{ Medication } \\
\hline Aspirin, n (\%) & $209(26.0)$ & $202(25.4)$ & 0.789 & $87(28.2)$ & $94(27.0)$ & 0.724 & $109(83.8)$ & 257 (9I.I) & 0.048 \\
\hline ACEI/ARB, n (\%) & $|3|(16.3)$ & $143(18.0)$ & 0.369 & $50(16.2)$ & $54(15.5)$ & 0.802 & $59(45.4)$ & $138(48.9)$ & 0.379 \\
\hline Diuretics, n (\%) & $407(50.6)$ & $310(39.0)$ & $<0.001$ & $14 \mid(45.8)$ & 137 (39.4) & 0.098 & $56(43.1)$ & $73(25.9)$ & $<0.001$ \\
\hline \multicolumn{10}{|l|}{ Biochemical data } \\
\hline MAP, mmHg & $54.9 \pm 15.3$ & $58.7 \pm \mid 3.1$ & $<0.001$ & $55.0 \pm 15.8$ & $58.9 \pm 12.8$ & 0.001 & $87.8 \pm 13.4$ & $94.6 \pm 13.0$ & $<0.001$ \\
\hline Hemoglobin, $g / L$ & $101.6 \pm 18.4$ & $108.5 \pm 21.1$ & $<0.001$ & $101.3 \pm 20.5$ & $106.0 \pm 21.0$ & 0.004 & $|09.1 \pm 23.4|$ & $130.0 \pm 18.6$ & $<0.001$ \\
\hline eGFR, mL/min & $52.5 \pm 18.4$ & $81.2 \pm 18.1$ & $<0.001$ & $52.0 \pm 18.1$ & $76.7 \pm 23.9$ & $<0.001$ & $57.1 \pm 15.3$ & $86.3 \pm 10.7$ & $<0.001$ \\
\hline Total bilirubin, umol/L & $31.3 \pm 8.6$ & $16.3 \pm 7.8$ & $<0.001$ & $30.9 \pm 16.1$ & $14.2 \pm 3.6$ & $<0.001$ & $17.0 \pm 10.4$ & $17.5 \pm 10.4$ & 0.661 \\
\hline Bicarbonate, mmol/L & $24.4 \pm 5.2$ & $24.8 \pm 4.6$ & 0.075 & $24.1 \pm 4.9$ & $25.6 \pm 4.5$ & 0.175 & $22.7 \pm 4.5$ & $23.8 \pm 4.6$ & 0.022 \\
\hline SOFA, points & $5.9 \pm 1.4$ & $3.8 \pm 1.8$ & $<0.001$ & $6.0 \pm 2.6$ & $3.9 \pm 2.0$ & $<0.001$ & $6.1 \pm 2.1$ & $3.1 \pm 1.3$ & $<0.001$ \\
\hline \multicolumn{10}{|l|}{ Inflammation index } \\
\hline NLR, n (\%) & & & 0.020 & & & 0.018 & & & $<0.001$ \\
\hline$\geq 9.1$ & $34 I(42.4)$ & $292(36.7)$ & & $134(43.5)$ & $120(34.5)$ & & $49(37.7)$ & $43(15.2)$ & \\
\hline$<9.1$ & $463(57.6)$ & $503(63.3)$ & & $174(56.5)$ & $228(65.5)$ & & $81(62.3)$ & $239(84.8)$ & \\
\hline PLR, n (\%) & & & 0.049 & & & 0.430 & & & 0.431 \\
\hline$\geq 510$ & $104(12.9)$ & $78(9.8)$ & & $32(10.4)$ & $43(12.4)$ & & $\mathrm{I}(0.8)$ & $5(1.8)$ & \\
\hline$<510$ & $700(87.1)$ & $717(90.2)$ & & $276(89.6)$ & $305(87.6)$ & & $129(99.2)$ & $277(98.2)$ & \\
\hline MLR, n (\%) & & & $<0.001$ & & & $<0.001$ & & & 0.006 \\
\hline$\geq 0.7$ & $399(49.6)$ & $654(82.3)$ & & $152(49.4)$ & $69(19.8)$ & & $27(20.8)$ & $30(10.6)$ & \\
\hline$<0.7$ & $405(50.4)$ & |4| (17.7) & & $156(50.6)$ & $279(80.2)$ & & $103(79.2)$ & $252(89.4)$ & \\
\hline SII, n (\%) & & & 0.056 & & & 0.801 & & & 0.810 \\
\hline$\geq 28 \mid 3.8$ & $576(71.6)$ & $192(24.2)$ & & $77(25.0)$ & $90(25.9)$ & & $12(9.2)$ & $24(8.5)$ & \\
\hline$<2813.8$ & $228(28.4)$ & $603(75.8)$ & & $231(75.0)$ & $258(74.1)$ & & II $8(90.8)$ & $258(91.5)$ & \\
\hline ALRI, n (\%) & & & $<0.001$ & & & $<0.001$ & & & 0.044 \\
\hline$\geq 110.6$ & $296(36.8)$ & $102(12.8)$ & & 115 (37.3) & $33(9.5)$ & & $3(2.3)$ & $4(1.4)$ & \\
\hline$<110.6$ & $508(63.2)$ & $693(87.2)$ & & $193(62.7)$ & $315(90.5)$ & & $127(97.7)$ & 278 (98.6) & \\
\hline CAR, n (\%) & & & 0.085 & & & 0.902 & & & 0.517 \\
\hline$\geq 0.8$ & $86(10.7)$ & $65(8.2)$ & & $22(7.1)$ & $24(6.9)$ & & $26(20.0)$ & $35(12.4)$ & \\
\hline$<0.8$ & 718 (89.3) & 730 (91.8) & & $286(92.9)$ & $324(93.1)$ & & $104(90.0)$ & 247 (87.6) & \\
\hline
\end{tabular}

(Continued) 
Table I (Continued).

\begin{tabular}{|c|c|c|c|c|c|c|c|c|c|}
\hline \multirow{3}{*}{$\begin{array}{l}\text { Characteristics } \\
\text { GPS, n (\%) } \\
0\end{array}$} & \multicolumn{3}{|c|}{ Training Set $(n=1599)$} & \multicolumn{3}{|c|}{ Internal Validation Set $(n=656)$} & \multicolumn{3}{|c|}{ External Validation Set $(n=4 \mid 2)$} \\
\hline & & & $<0.001$ & & & $<0.001$ & & & $<0.001$ \\
\hline & $108(13.4)$ & $197(24.8)$ & & $38(12.3)$ & $87(25.0)$ & & $44(33.8)$ & $160(56.7)$ & \\
\hline I & $46 \mathrm{I}(57.3)$ & $416(52.3)$ & & I8I (58.8) & $187(53.7)$ & & $58(44.6)$ & $107(37.9)$ & \\
\hline 2 & $235(29.3)$ & $182(22.9)$ & & $89(28.9)$ & $74(21.3)$ & & $28(21.5)$ & $15(5.3)$ & \\
\hline mGPS, n (\%) & & & 0.006 & & & 0.017 & & & $<0.001$ \\
\hline 0 & $537(66.8)$ & $576(72.5)$ & & $204(66.2)$ & $260(74.7)$ & & $74(57.0)$ & $206(73.0)$ & \\
\hline I & $32(4.0)$ & $37(4.7)$ & & $15(4.9)$ & $14(4.0)$ & & $28(21.5)$ & $61(21.6)$ & \\
\hline 2 & $235(29.2)$ & $182(22.9)$ & & $89(28.9)$ & $74(21.3)$ & & $28(21.5)$ & $15(5.3)$ & \\
\hline PI, n (\%) & & & 0.042 & & & 0.037 & & & $<0.001$ \\
\hline 0 & $310(38.6)$ & $338(42.5)$ & & $123(39.9)$ & $158(45.4)$ & & $54(41.5)$ & $164(58.2)$ & \\
\hline I & $362(45.0)$ & $347(43.6)$ & & $125(40.6)$ & I44 (4I.4) & & $55(42.3)$ & $98(34.8)$ & \\
\hline 2 & $132(16.4)$ & $110(13.8)$ & & $60(19.5)$ & $46(13.2)$ & & $21(16.2)$ & $20(7.1)$ & \\
\hline \multicolumn{10}{|l|}{ Outcomes, n (\%) } \\
\hline AKI & & & - & & & - & & & - \\
\hline Stage I & $446(55.5)$ & - & - & I7I (55.5) & - & - & $72(55.4)$ & - & - \\
\hline Stage 2 & $153(19.0)$ & - & - & $61(19.8)$ & - & - & $37(28.5)$ & - & - \\
\hline Stage 3 & $205(25.5)$ & - & - & $76(24.7)$ & - & - & $21(16.1)$ & - & - \\
\hline In-hospital mortality & $\mid 72(2 \mid .4)$ & $54(6.8)$ & $<0.001$ & $60(19.5)$ & $37(10.6)$ & 0.001 & $44(33.8)$ & $17(6.0)$ & $<0.001$ \\
\hline 2-year mortality, n (\%) & $405(50.4)$ & $237(29.8)$ & $<0.001$ & $153(49.7)$ & $137(39.4)$ & $<0.001$ & $70(53.8)$ & $42(14.9)$ & $<0.001$ \\
\hline
\end{tabular}

Abbreviations: AKI, acute kidney disease; BMI, body mass index; ACS, acute coronary syndrome; CKD, chronic kidney disease; ACEI/ARB, angiotensin converting enzyme inhibitors/angiotensin receptor blocker; MAP, mean arterial pressure; eGFR, estimated glomerular filtration rate; SOFA, sequential organ failure assessment; NLR, neutrophil-lymphocyte ratio; PLR, platelet-lymphocyte ratio; MLR, monocyte-lymphocyte ratio; SII, systemic immune-inflammation index; ALRI, aspartate aminotransferase to lymphocyte ratio; CAR, C-reactive protein-albumin ratio; GPS, Glasgow prognostic score; mGPS, modified Glasgow prognostic score; PI, Prognostic index.

Table 2 Logistic Regression Models for AKI of Laboratory Parameters in the Test Cohort

\begin{tabular}{|l|l|l|l|l|l|l|}
\hline \multirow{2}{*}{} & \multicolumn{3}{|l|}{ Univariate } & \multicolumn{2}{l|}{ Multivariate } \\
\cline { 2 - 7 } & $\boldsymbol{\beta}$ & OR & $\mathbf{P}$ & $\boldsymbol{\beta}$ & $\mathbf{O R}$ & $\mathbf{P}$ \\
\hline NLR & 0.015 & 1.015 & 0.008 & 0.035 & 1.028 & 0.063 \\
PLR & 0.000 & 1.000 & 0.350 & & & \\
Ig (PLR) & 0.057 & 1.059 & 0.694 & & & \\
MLR & 1.938 & 6.942 & $<0.001$ & 2.137 & 8.470 & $<0.001$ \\
SII & 0.000 & 1.000 & 0.191 & & & \\
Ig (SII) & 0.084 & 1.087 & 0.470 & & & \\
ALRI & 0.010 & 1.010 & $<0.001$ & 0.007 & 1.007 & $<0.001$ \\
CAR & 0.426 & 1.530 & 0.011 & -0.163 & 0.850 & 0.653 \\
GPS & 0.401 & 1.494 & $<0.001$ & 0.417 & 1.518 & 0.006 \\
mGPS & 0.157 & 1.170 & 0.006 & -0.163 & 0.839 & 0.266 \\
PI & 0.133 & 1.142 & 0.023 & 0.074 & 1.077 & 0.539 \\
\hline
\end{tabular}

Abbreviations: AKI, acute kidney injury; OR, odds ratio; NLR, neutrophillymphocyte ratio; PLR, platelet-lymphocyte ratio; MLR, monocyte-lymphocyte ratio; SII, systemic immune-inflammation index; ALRI, aspartate aminotransferase to lymphocyte ratio; CAR, C-reactive protein-albumin ratio; CPAR, C-reactive protein-prealbumin ratio; GPS, Glasgow prognostic score; mGPS, modified Glasgow prognostic score; PI, Prognostic Index.
AKI in the training set, internal validation set, and in the external validation set were in good agreement. Hence, the nomogram was well calibrated.

\section{Construction and Validation of a Survival Nomogram}

As shown in Table 4, we selected four variables from multivariate Cox analysis (age, ASII, SOFA scores, hypertension, diabetes, and HGB) into the survival nomogram to evaluate the 2-year mortality (Figure 2B). Moreover, td-ROC analysis was also used to evaluate the predictive efficiency of the nomogram in predicting 1-month, 1-year, and 2-year OS. In the assessment of 1-month, 1-year, and 2-year survival rates, the predictive power of the survival nomogram, measured by AUCs, were 0.852, 0.811, and 0.789 in the training set (Figure 4A); 0.883, 0.842, and 0.821 in the internal validation set (Figure 4B); and $0.775,0.727$, and 0.750 in the external validation set, respectively (Figure 4C). 
Table 3 Multivariate Logistic Regression Analyses of ASII Scores for AKI

\begin{tabular}{|c|c|c|c|c|c|c|}
\hline \multirow[t]{2}{*}{ ASII Scores } & \multicolumn{2}{|l|}{ Training Set } & \multicolumn{2}{|l|}{ Internal Validation Set } & \multicolumn{2}{|l|}{ External Validation set } \\
\hline & Adjusted $\mathrm{OR}^{\mathrm{a}}(95 \% \mathrm{Cl})$ & $P$ value & Adjusted OR $(95 \% \mathrm{Cl})$ & $P$ value & Adjusted OR (95\% Cl) & $P$ value \\
\hline \multicolumn{7}{|l|}{ All study participants } \\
\hline Low & Ref. & - & Ref. & - & Ref. & - \\
\hline Intermediate & $4.25(3.25-5.56)$ & $<0.001$ & $4.17(2.76-6.32)$ & $<0.001$ & $2.38(0.97-4.83)$ & 0.058 \\
\hline High & $5.33(3.60-7.88)$ & $<0.001$ & $6.09(3.32-11.16)$ & $<0.001$ & $3.09(\mid .04-5.31)$ & 0.039 \\
\hline \multicolumn{7}{|l|}{ Patients with CKD } \\
\hline Low & Ref. & - & Ref. & - & Ref. & - \\
\hline Intermediate & $2.76(1.32-9.70)$ & 0.013 & $6.38(1.89-21.55)$ & 0.003 & $0.77(0.05-11.20)$ & 0.850 \\
\hline High & $3.98(1.87-8.47)$ & $<0.001$ & $8.69(1.31-57.76)$ & 0.025 & $1.94(0.04-89.39)$ & 0.736 \\
\hline \multicolumn{7}{|l|}{ Patients without CKD } \\
\hline Low & Ref. & - & Ref. & - & Ref. & - \\
\hline Intermediate & $4.59(3.4 I-6.17)$ & $<0.001$ & $4.26(2.70-6.72)$ & $<0.001$ & $2.23(0.84-5.97)$ & 0.110 \\
\hline High & $5.51(3.58-8.47)$ & $<0.001$ & $6.23(3.24-11.98)$ & $<0.001$ & $3.23(1.05-5.74)$ & 0.038 \\
\hline
\end{tabular}

Notes: ${ }^{2}$ Adjusted for age, gender, BMI, hypertension, diabetes, preexisting CKD, mean arterial pressure, hemoglobin, SOFA score, serum albumin, total bilirubin, eGFR, treatment with loop diuretics, treatment with ACEI/ARB, and treatment with aspirin. AKI: Age+T2DM+eGFR+SOFA+HGB+ASII. Mortalityge+HTN+T2DM+SOFA+ASII + HGB.

Abbreviations: OR, odds ratio; $95 \% \mathrm{Cl}, 95 \%$ confidence interval.

Furthermore, the calibration curves (Figure 3D-I) for the survival nomogram demonstrated that the predicted survival rates calculated by the survival nomogram were highly consistent with the actual values, indicating that the survival nomogram for OS had reliable repeatability.

\section{Discussion}

In the present study, we aimed to create a novel inflammation-based index incorporating significant hematological results to expand the existing fragmented knowledge regarding systemic inflammation. To the best of our knowledge, this is the first study to demonstrate the predictive importance of the combined score (ASII), which is specific to AKI. The ASII showed a relatively better risk stratification for AKI in ICU patients. Moreover, the nomograms integrating ASII and other significant clinical variables reached a much higher predictive ability in predicting AKI and 2-year mortality in ICU patients than any other single index. To further validate
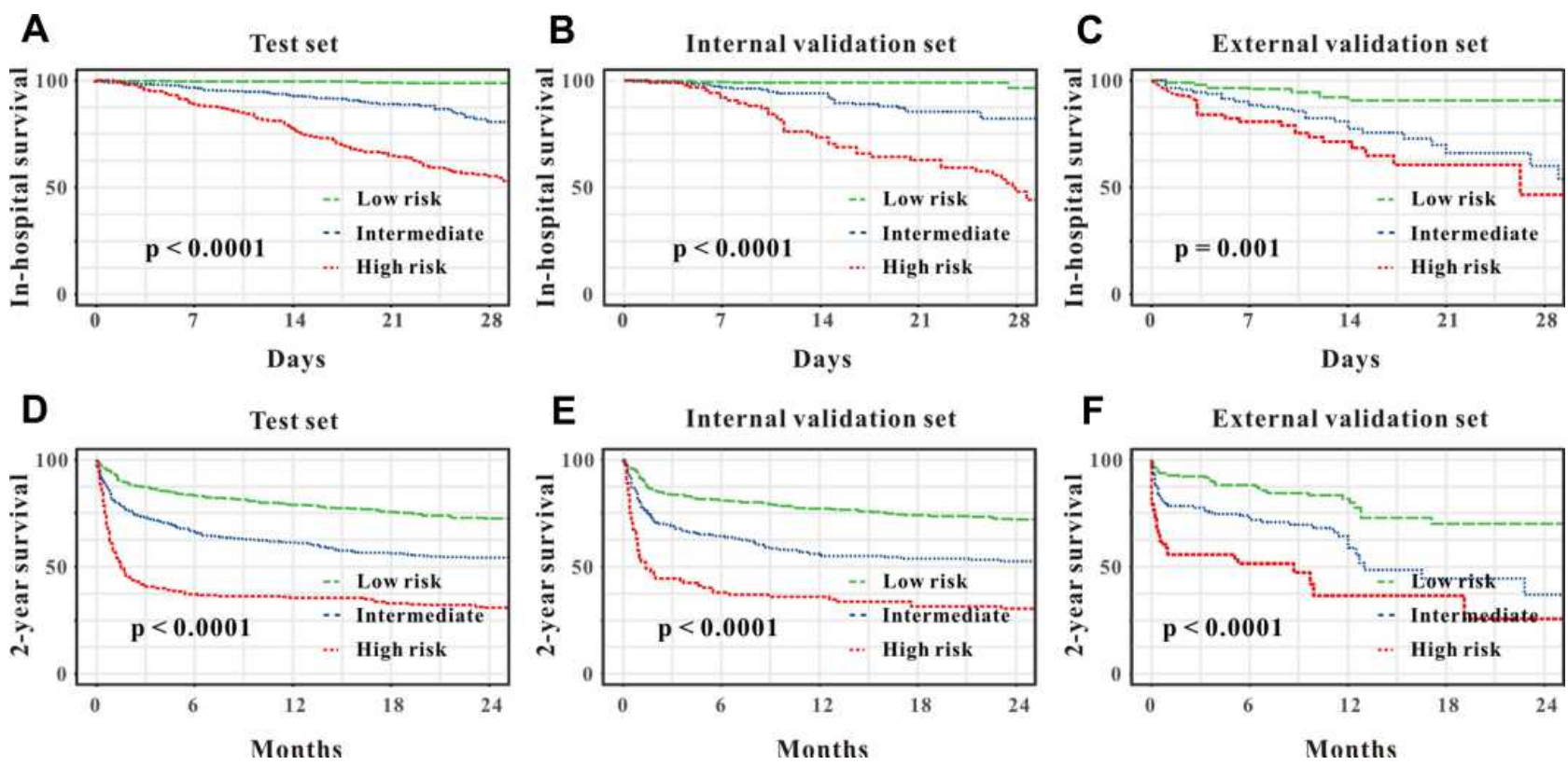

Figure I Kaplan-Meier curves for in-hospital survival for all patients stratified by ASIl in the test set (A), in the internal validation ser (B), and in the external validation set (C). Kaplan-Meier curves for 2-year survival for all patients stratified by ASIl in the test set (D), in the internal validation ser (E), and in the external validation set (F). 


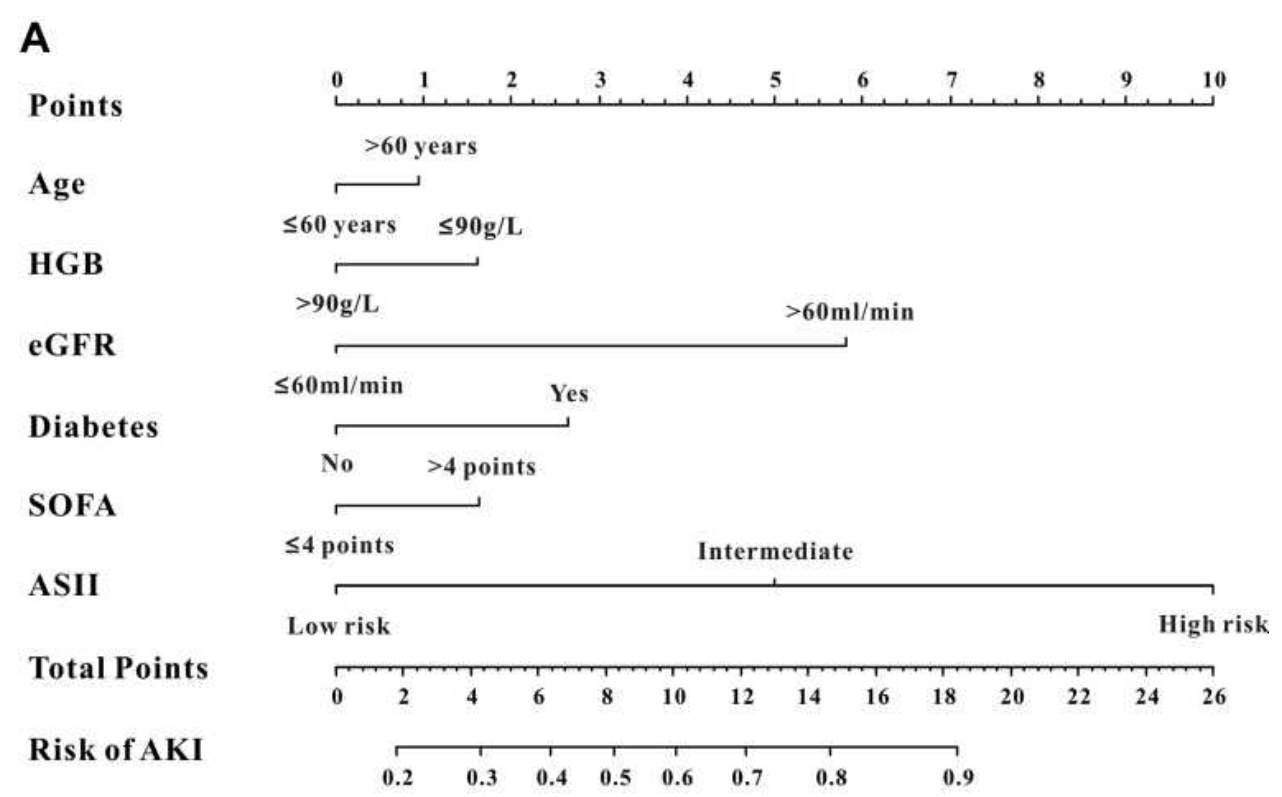

B

Points

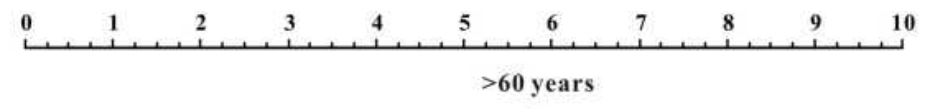

Age

SOFA

Hypertension

Diabetes

HGB

ASII

Total Points
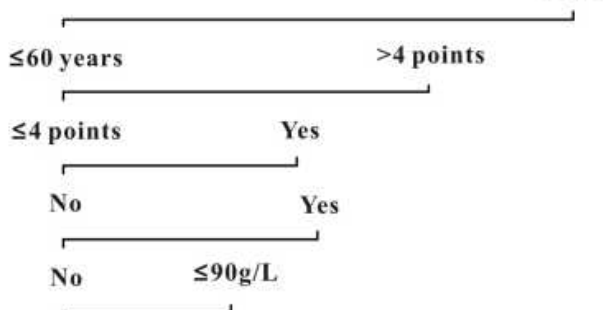

$>90 \mathrm{~g} / \mathrm{L} \quad$ Intermediate

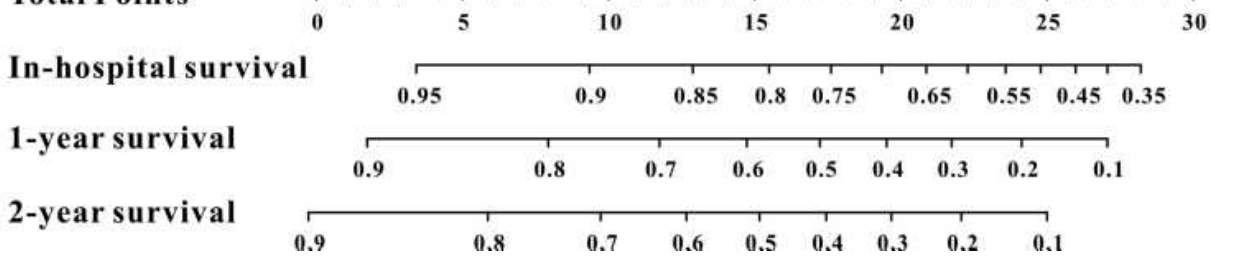

Figure 2 Evaluation of AKI (A) and 2-year mortality (B) associated nomograms for ICU patients.

the feasibility of the predictive value of the nomograms incorporating ASII and other clinical variables, we independently verified this conclusion in our $\mathrm{CCU}$ patients. Therefore, these data suggest that the ASII may be a good predictor for identifying patients at high risk of AKI and mortality among ICU patients.

AKI is a common clinical complication due to pathogenic conditions, such as ischemic and toxic insults. ${ }^{19}$ In the past decades, an increasing number of studies have demonstrated the role of inflammation in the physiopathology of AKI. ${ }^{20,21}$ Locally, ischemia or reperfusion initiates changes in renal tubular epithelial cells, endothelial cells, and leukocytes, resulting in the loss of immunological homeostasis in the kidney. Subsequent inflammation leads to parenchymal cell death and, in severe cases, AKI. ${ }^{22,23}$ In addition, AKI may be caused by generalized systemic inflammation due to increased renal production, reduced renal clearance, or impaired metabolism of inflammatory mediators. ${ }^{24}$ Importantly, recent 

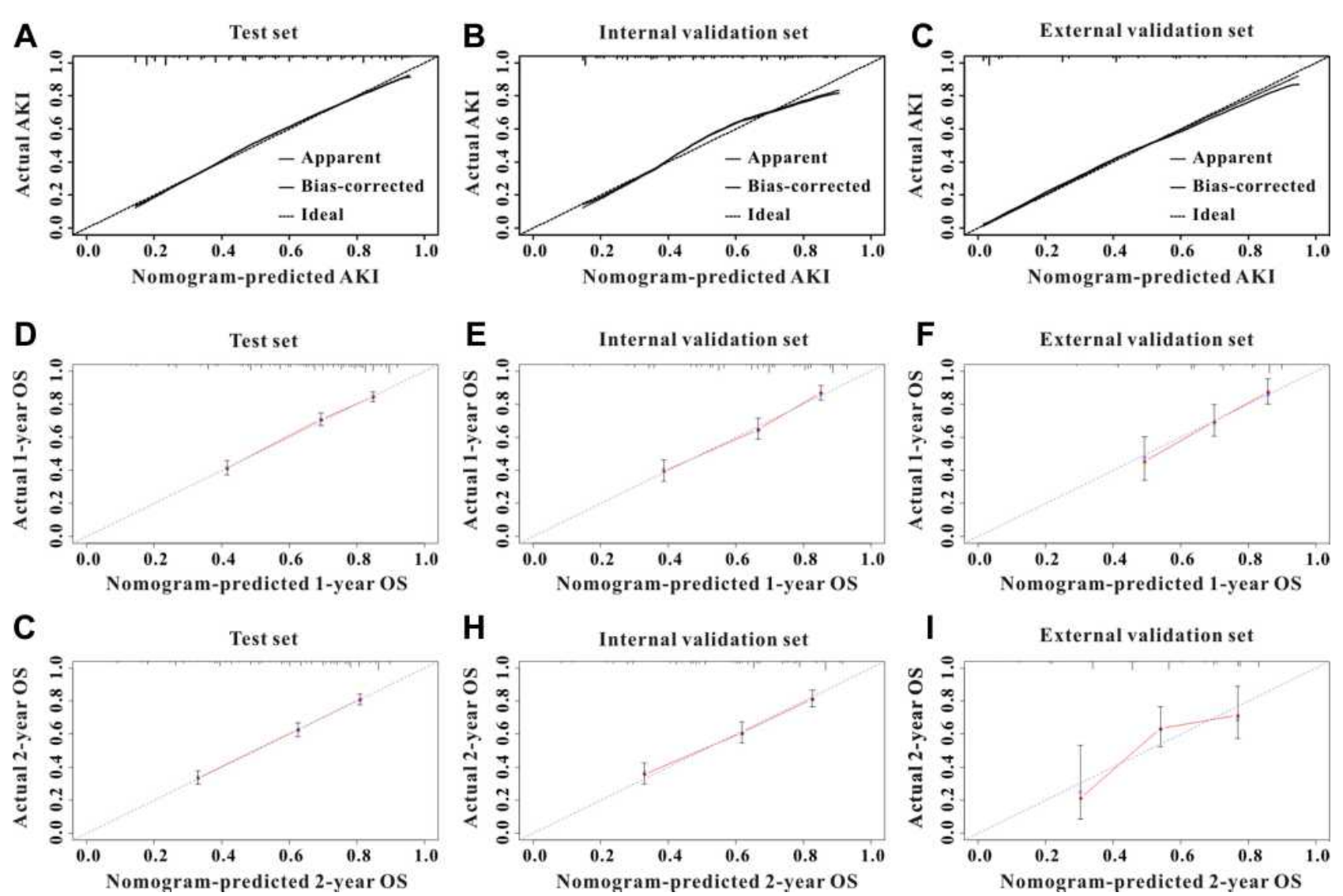

Figure 3 Calibration curves for AKI and mortality for ICU patients. The calibration curves for predicting AKI in the training set (A), in the internal validation set (B), and in the external validation set (C). The calibration curves for predicting all-cause mortality at I-year (D) and 2-year (G) in the training set, and I-year (E) and 2-year (H) in the internal validation set, and I-year (F) and 2-year (I) in the external validation set.

studies have found that AKI can lead to long-term injury and cause renal fibrosis and chronic inflammation, both of which are common features of CKD. ${ }^{25,26}$ In contrast, the progression of AKI or CKD can be prevented and delayed by suppressing the inflammatory response. Angiotensin II has been shown to trigger reactive oxygen species, activate nuclear factor kappa $\mathrm{B}$, and augment tumor necrosis alpha production from macrophages. ${ }^{27}$ ACEIs and ARBs can suppress tumor necrosis alpha production and prevent nuclear factor kappa $\mathrm{B}$ translocation to the nucleus to provide hemodynamicindependent renoprotection. ${ }^{28}$ In a previous study, losartan, one of the most widely used ARBs, could provide effective renoprotection and anti-inflammatory effects in experimental diabetic nephropathy ${ }^{29}$ and these beneficial effects could be remarkably magnified even after advanced kidney injury when losartan was administered along with hydrochlorothiazide. ${ }^{30}$ In a prospective clinical trial of 52 hemodialysis patients and 30 age- and sex-matched healthy volunteers, Kayabasi et al concluded that losartan treatment could prevent differentiation of oxidative stress-related inflammation. ${ }^{31}$ Thus, there is an interaction between AKI, inflammatory mediators, and CKD.
In this study, we created a novel inflammation-based index incorporating hematological factors of AKI and found that it could serve as a good risk stratification marker for AKI and prognosis in patients in the ICU.

The MLR is a predictor of the patient's response to inflammatory injury, and the association between the MLR and prognosis in patients from different populations has been well studied in previous studies. ${ }^{32,33}$ In a retrospective study of 678 patients with non-ST elevated myocardial infarction who underwent primary percutaneous coronary intervention, Fan et al concluded that the MLR was an independent predictor of long-term major adverse cardiac events (HR, 2.22; 95\% CI, 1.46-3.11). ${ }^{34}$ A similar result was also found in another study. ${ }^{35}$ Our study investigated the prognostic value of the MLR in patients in the ICU. Moreover, we further combined the MLR and two inflammatory biomarkers (the GPS and ALRI) to establish an ideal inflammatory predictor specific to AKI, and the ASII score had an excellent prognostic value for critically ill patients. 
Table 4 Cox Proportional Hazards Analysis of the Ability of ASIl for Secondary Outcomes in Training Set

\begin{tabular}{|c|c|c|c|c|}
\hline & \multicolumn{2}{|l|}{ Univariate } & \multicolumn{2}{|l|}{ Multivariate } \\
\hline & HR (95\% Cl) & $P$-value & HR $(95 \% \mathrm{Cl})$ & $P$-value \\
\hline \multicolumn{5}{|l|}{ For in-hospital mortality } \\
\hline ASII score & $1.25(1.21-1.28)$ & $<0.001$ & $1.19(1.15-1.23)$ & $<0.001$ \\
\hline Age, years & $1.04(1.04-1.05)$ & $<0.001$ & $1.03(1.02-1.05)$ & $<0.001$ \\
\hline Male (versus female) & $1.21(0.92-1.58)$ & 0.169 & & \\
\hline BMI, $\mathrm{kg} / \mathrm{m}^{2}$ & $0.97(0.96-1.02)$ & 0.153 & & \\
\hline Preexisting CKD & $1.21(0.90-1.63)$ & 0.209 & & \\
\hline Hypertension & $1.32(0.98-1.78)$ & 0.072 & & \\
\hline Diabetes & $1.33(1.01-1.75)$ & 0.039 & $1.29(0.96-1.74)$ & 0.091 \\
\hline Aspirin & $0.85(0.62-1.17)$ & 0.323 & & \\
\hline ACEI/ARB & $0.68(0.45-1.02)$ & 0.060 & & \\
\hline Diuretics & $0.98(0.76-1.28)$ & 0.898 & & \\
\hline MAP, $\mathrm{mmHg}$ & $0.98(0.97-0.99)$ & $<0.001$ & $0.99(0.98-0.99)$ & 0.029 \\
\hline Hemoglobin, g/L & 1.01 (1.00-1.02) & 0.041 & $1.01(1.00-1.02)$ & 0.008 \\
\hline eGFR, mL/min & $0.98(0.97-0.99)$ & $<0.001$ & $1.00(1.00-1.01)$ & 0.264 \\
\hline Total bilirubin, umol/L & 1.01 (1.00-1.02) & $<0.001$ & $1.00(1.00-1.01)$ & 0.078 \\
\hline Bicarbonate, $\mathrm{mmol} / \mathrm{L}$ & $0.92(0.90-0.95)$ & $<0.001$ & $0.94(0.91-0.97)$ & $<0.001$ \\
\hline SOFA, points & $1.14(1.11-1.18)$ & $<0.001$ & $1.08(1.04-1.12)$ & $<0.001$ \\
\hline \multicolumn{5}{|l|}{ For 2-year mortality } \\
\hline ASII score & $1.19(1.16-1.21)$ & $<0.001$ & $1.14(1.11-1.17)$ & $<0.001$ \\
\hline Age, years & $1.03(1.02-1.03)$ & $<0.001$ & $1.03(1.02-1.03)$ & $<0.001$ \\
\hline Male (versus female) & $1.24(1.06-1.45)$ & 0.008 & I.I8 (0.99-1.39) & 0.055 \\
\hline BMI, $\mathrm{kg} / \mathrm{m}^{2}$ & $0.99(0.98-1.00)$ & 0.263 & & \\
\hline Preexisting CKD & $2.00(1.69-2.36)$ & $<0.001$ & $1.15(0.92-1.44)$ & 0.216 \\
\hline Hypertension & $1.38(1.17-1.64)$ & $<0.001$ & $1.29(1.06-1.57)$ & 0.011 \\
\hline Diabetes & $1.67(1.43-1.96)$ & $<0.001$ & $1.39(1.16-1.65)$ & $<0.001$ \\
\hline Aspirin & $0.96(0.80-1.14)$ & 0.620 & & \\
\hline ACEI/ARB & $0.88(0.7 \mathrm{I}-1.08)$ & 0.227 & & \\
\hline Diuretics & 1.19 (1.02-1.39) & 0.031 & $0.95(0.81-1.12)$ & 0.529 \\
\hline MAP, $\mathrm{mmHg}$ & $0.98(0.97-0.99)$ & $<0.001$ & $1.00(0.99-1.01)$ & 0.759 \\
\hline Hemoglobin, g/L & $0.98(0.98-0.99)$ & $<0.001$ & $0.99(0.98-0.99)$ & 0.003 \\
\hline eGFR, mL/min & $0.99(0.98-0.99)$ & $<0.001$ & $1.00(1.00-1.01)$ & 0.331 \\
\hline Total bilirubin, umol/L & 1.01 (1.00-1.01) & $<0.001$ & $1.00(0.99-1.01)$ & 0.202 \\
\hline Bicarbonate, $\mathrm{mmol} / \mathrm{L}$ & $0.99(0.97-1.01)$ & 0.203 & I.0I (0.89-I.40) & 0.506 \\
\hline SOFA, points & $1.14(1.11-1.16)$ & $<0.001$ & $1.08(1.05-1.11)$ & $<0.001$ \\
\hline
\end{tabular}

Abbreviations: HR, hazard ratio; $95 \% \mathrm{Cl}, 95 \%$ confidence interval; ASII, AKI specific inflammatory index; BMI, body mass index; CKD, chronic kidney disease; ACEI/ARB, angiotensin converting enzyme inhibitors/angiotensin receptor blocker; MAP, mean arterial pressure; eGFR, estimated glomerular filtration rate; SOFA, sequential organ failure assessment.

A nomogram, which is an easy-to-use predictive tool, can quantify risk by developing a visualized image and has been widely applied in clinical practice. ${ }^{36,37}$ A nomogram incorporating some important characteristics is an intuitive and easily accessible instrument for clinicians to definitively diagnose and determine the prognosis for different sets of patients. ${ }^{38,39}$ In the present study, we successfully created two nomograms based on ASII scores and other informative markers to predict the incidence of AKI and 2-year survival in patients in the ICU. Furthermore, independent validation of the two nomograms is vital to clarify the bias of the assessment in different individuals, evaluate its applicability to different study populations, and thus increase the confidence of the nomogram. In this study, we independently validated two nomograms in patients in our hospital, with both nomograms achieving good predictive abilities in the validation set.

Despite the relatively large sample size, four limitations were observed in this study. First, although we collected the hematological results of each patient on ICU admission, values of inflammatory indices may vary over time, and dynamic monitoring of these inflammatory 

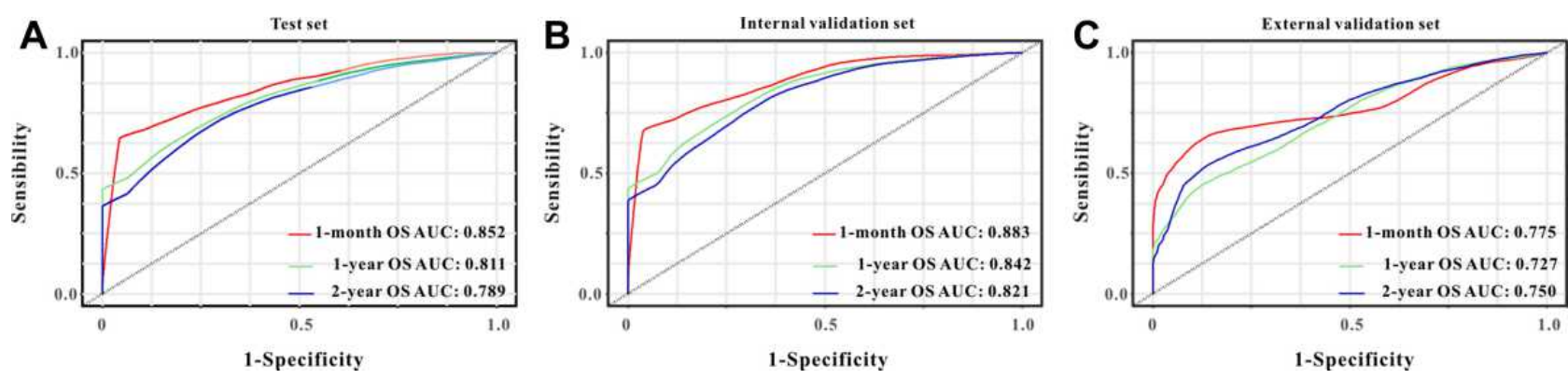

Figure 4 Time-dependent ROC curves by 2-year survival associated nomogram for predicting I-month, I-year, and 2-year survival rates in the training (A) set, in the internal validation set $(\mathbf{B})$, and in the external validation set $(\mathbf{C})$.

indices may be more accurate. Second, we did not obtain levels of serum cytokines, such as interleukin and tumor necrosis factor. Third, in the validation cohort, only a small fraction of ICU patients had data on CRP levels, and there may have been bias after excluding them. Lastly, the impact of gene profiling related to inflammatory pathways was not assessed due to the lack of related medical records. Therefore, large-scale clinical studies assessing more clinical and genetic features are needed to validate the conclusions of our study.

\section{Conclusion}

We developed a novel inflammatory index based on inflammatory indices significantly associated with the incidence of AKI and prognosis in patients in the ICU. The ASII showed a relatively better risk stratification for AKI in ICU patients than other inflammatory indices. Moreover, the nomograms integrating the ASII and other significant clinical variables had a much high predictive ability in predicting AKI and 2-year mortality in ICU patients. However, larger scale prospective trials are warranted to validate the results of our study.

\section{Data Sharing Statement}

All data in our study are available from the corresponding author of prof. Lu upon reasonable request.

\section{Statement of Ethics}

Access to the MIMIC-III database for research was approved by the Institutional Review Boards of the Massachusetts Institute of Technology (Cambridge, MA, USA) and the Beth Israel Deaconess Medical Center and was granted a waiver of informed consent. Due to the HIPAA-compliant deidentification in the MIMIC-III database, our institutional IRB requirement and informed consent for this study were waived. In summary, the study was performed in accordance with the ethical standards laid down in the 1964 Declaration of Helsinki and its later amendments. Moreover, this study was also approved by the Ethics Committee of Zhongnan hospital of Wuhan university.

\section{Funding}

There is no funding to report.

\section{Disclosure}

The authors declare that they have no competing interests.

\section{References}

1. Hoste E, Kellum JA, Selby NM, et al. Global epidemiology and outcomes of acute kidney injury. Nat Rev Nephrol. 2018;14:607-625. doi:10.1038/s41581-018-0052-0

2. Kellum JA. Why are patients still getting and dying from acute kidney injury? Curr Opin Crit Care. 2016;22:513-519. doi:10.1097/ MCC. 0000000000000358

3. Mehta RL, Cerda J, Burdmann EA, et al. International Society of Nephrology's 0by 25 initiative for acute kidney injury (zero preventable deaths by 2025): a human rights case for nephrology. Lancet. 2015;385:2616-2643. doi:10.1016/S0140-6736(15)60126-X

4. Collister D, Pannu N, Ye F, et al. Health care costs associated with AKI. Clin J Am Soc Nephrol. 2017;12:1733-1743. doi:10.2215/ CJN.00950117

5. Hobson C, Ozrazgat-Baslanti T, Kuxhausen A, et al. Cost and mortality associated with postoperative acute kidney injury. Ann Surg. 2015;261:1207-1214. doi:10.1097/SLA.0000000000000732

6. Tanik VO, Cinar T, Velibey Y, et al. Neutrophil-to-lymphocyte ratio predicts contrast-induced acute kidney injury in patients with ST-elevation myocardial infarction treated with primary percutaneous coronary intervention. J Tehran Heart Cent. 2019;14:59-66.

7. Abu AM, Slotki I, Shavit L. Single emergency room measurement of neutrophil/lymphocyte ratio for early detection of acute kidney injury (AKI). Intern Emerg Med. 2018;13:717-725. doi:10.1007/s11739-0171715-8

8. Velibey Y, Oz A, Tanik O, et al. Platelet-to-lymphocyte ratio predicts contrast-induced acute kidney injury in patients with ST-segment elevation myocardial infarction undergoing primary percutaneous coronary intervention. Angiology. 2017;68:419-427. doi:10.1177/ 0003319716660244 
9. Zheng C-F, Liu W-Y, Zeng -F-F, et al. Prognostic value of platelet-tolymphocyte ratios among critically ill patients with acute kidney injury. Crit Care. 2017;21(1):238. doi:10.1186/s13054-017-1821-z

10. Karabag Y, Cagdas M, Rencuzogullari I, et al. The C-reactive protein to albumin ratio predicts acute kidney injury in patients with ST-segment elevation myocardial infarction undergoing primary percutaneous coronary intervention. Heart Lung Circ. 2019;28:1638-1645. doi:10.1016/j.hlc.2018.08.009

11. Johnson AE, Pollard TJ, Shen L, et al. MIMIC-III, a freely accessible critical care database. Sci Data. 2016;3:160035. doi:10.1038/ sdata.2016.35

12. Hu Y, Liu H, Fu S, Wan J, Li X. Red blood cell distribution width is an independent predictor of AKI and mortality in patients in the coronary care unit. Kidney Blood Press Res. 2017;42:1193-1204. doi:10.1159/000485866

13. Camp RL, Dolled-Filhart M, Rimm DL. X-tile: a new bio-informatics tool for biomarker assessment and outcome-based cut-point optimization. Clin Cancer Res. 2004;10:7252-7259. doi:10.1158/ 1078-0432.CCR-04-0713

14. Park JH, Watt DG, Roxburgh CS, Horgan PG, McMillan DC. Colorectal cancer, systemic inflammation, and outcome: staging the tumor and staging the host. Ann Surg. 2016;263:326-336. doi:10.1097/SLA.0000000000001122

15. Li Z, Li S, Ying X, et al. The clinical value and usage of inflammatory and nutritional markers in survival prediction for gastric cancer patients with neoadjuvant chemotherapy and D2 lymphadenectomy. Gastric Cancer. 2020;23:540-549. doi:10.1007/s10120-019-01027-6

16. Kellum JA, Lameire N. Diagnosis, evaluation, and management of acute kidney injury: a KDIGO summary (Part 1). Crit Care. 2013;17:204. doi:10.1186/cc11454

17. McCullough PA, Shaw AD, Haase M, et al. Diagnosis of acute kidney injury using functional and injury biomarkers: workgroup statements from the tenth Acute Dialysis Quality Initiative Consensus Conference. Contrib Nephrol. 2013;182:13-29.

18. Levey AS, Stevens LA, Schmid $\mathrm{CH}$, et al. A new equation to estimate glomerular filtration rate. Ann Intern Med. 2009;150:604-612. doi:10.7326/0003-4819-150-9-200905050-00006

19. Sato Y, Yanagita M. Immune cells and inflammation in AKI to CKD progression. Am J Physiol Renal Physiol. 2018;315:F1501-12.

20. Bonventre JV, Yang L. Cellular pathophysiology of ischemic acute kidney injury. J Clin Invest. 2011;121:4210-4221. doi:10.1172/ JCI45161

21. Sharfuddin AA, Molitoris BA. Pathophysiology of ischemic acute kidney injury. Nat Rev Nephrol. 2011;7:189-200. doi:10.1038/ nrneph.2011.16

22. Li L, Huang L, Sung SS, et al. NKT cell activation mediates neutrophil IFN-gamma production and renal ischemia-reperfusion injury. J Immunol. 2007;178:5899-5911. doi:10.4049/jimmunol.178.9.5899

23. Day YJ, Huang L, Ye H, Li L, Linden J, Okusa MD. Renal ischemia-reperfusion injury and adenosine $2 \mathrm{~A}$ receptor-mediated tissue protection: the role of CD4+ T cells and IFN-gamma. J Immunol. 2006;176:3108-3114. doi:10.4049/jimmunol.176.5.3108

24. Hoke TS, Douglas IS, Klein CL, et al. Acute renal failure after bilateral nephrectomy is associated with cytokine-mediated pulmonary injury. J Am Soc Nephrol. 2007;18:155-164. doi:10.1681/ ASN.2006050494

25. Chawla LS, Kimmel PL. Acute kidney injury and chronic kidney disease: an integrated clinical syndrome. Kidney Int. 2012;82:516-524. doi:10.1038/ki.2012.208
26. Chevalier RL. The proximal tubule is the primary target of injury and progression of kidney disease: role of the glomerulotubular junction. Am J Physiol Renal Physiol. 2016;311:F145-61. doi:10.1152/ ajprenal.00164.2016

27. Dandona P, Dhindsa S, Ghanim H, Chaudhuri A. Angiotensin II and inflammation: the effect of angiotensin-converting enzyme inhibition and angiotensin II receptor blockade. J Hum Hypertens. 2007;21:20-27. doi:10.1038/sj.jhh.1002101

28. Mizushima T, Sasaki M, Ando T, et al. Blockage of angiotensin II type 1 receptor regulates TNF-alpha-induced MAdCAM-1 expression via inhibition of NF-kappaB translocation to the nucleus and ameliorates colitis. Am J Physiol Gastrointest Liver Physiol. 2010;298: G255-66. doi:10.1152/ajpgi.00264.2009

29. Teles F, Machado FG, Ventura BH, et al. Regression of glomerular injury by losartan in experimental diabetic nephropathy. Kidney Int. 2009;75:72-79. doi:10.1038/ki.2008.528

30. Arias SC, Valente CP, Machado FG, et al. Regression of albuminuria and hypertension and arrest of severe renal injury by a losartan-hydrochlorothiazide association in a model of very advanced nephropathy. PLoS One. 2013;8:e56215. doi:10.1371/journal. pone.0056215

31. Kayabasi H, Yilmaz Z, Sit D, Kadiroglu AK, Yilmaz E. The effects of Losartan on oxidative stress and inflammation in non-diabetic patients undergoing chronic hemodialysis. Eur Rev Med Pharmacol Sci. 2013;17:235-242.

32. Shi L, Qin X, Wang H, et al. Elevated neutrophil-to-lymphocyte ratio and monocyte-to-lymphocyte ratio and decreased platelet-tolymphocyte ratio are associated with poor prognosis in multiple myeloma. Oncotarget. 2017;8:18792-18801. doi:10.18632/ oncotarget. 13320

33. Cananzi F, Minerva EM, Sama L, et al. Preoperative monocyte-tolymphocyte ratio predicts recurrence in gastrointestinal stromal tumors. J Surg Oncol. 2019;119:12-20. doi:10.1002/jso.25290

34. Fan Z, Li Y, Ji H, Jian X. Prognostic utility of the combination of monocyte-to-lymphocyte ratio and neutrophil-to-lymphocyte ratio in patients with NSTEMI after primary percutaneous coronary intervention: a retrospective cohort study. BMJ Open. 2018;8:e23459. doi:10.1136/bmjopen-2018-023459

35. Chen H, Li M, Liu L, Dang X, Zhu D, Tian G. Monocyte/lymphocyte ratio is related to the severity of coronary artery disease and clinical outcome in patients with non-ST-elevation myocardial infarction. Medicine (Baltimore). 2019;98:e16267. doi:10.1097/ MD.0000000000016267

36. Gittleman H, Sloan AE, Barnholtz-Sloan JS. An independently validated survival nomogram for lower-grade glioma. Neuro Oncol. 2020;22:665-674. doi:10.1093/neuonc/noz191

37. Liao F, Guo X, Lu X, Dong W. A validated survival nomogram for early-onset diffuse gastric cancer. Aging (Albany NY). 2020;12:13160-13171. doi:10.18632/aging.103406

38. He Y, Zhu Z, Chen Y, et al. Development and validation of a novel diagnostic nomogram to differentiate between intestinal tuberculosis and crohn's disease: a 6-year Prospective Multicenter Study. Am J Gastroenterol. 2019;114:490-499. doi:10.14309/ ajg.0000000000000064

39. Jiang Y, Yuan Q, Lv W, et al. Radiomic signature of (18) F fluorodeoxyglucose PET/CT for prediction of gastric cancer survival and chemotherapeutic benefits. Theranostics. 2018;8:5915-5928. doi: $10.7150 /$ thno. 28018 


\section{Publish your work in this journal}

The International Journal of General Medicine is an international, peer-reviewed open-access journal that focuses on general and internal medicine, pathogenesis, epidemiology, diagnosis, monitoring and treatment protocols. The journal is characterized by the rapid reporting of reviews, original research and clinical studies across all disease areas. The manuscript management system is completely online and includes a very quick and fair peer-review system, which is all easy to use. Visit http://www.dovepress.com/ testimonials.php to read real quotes from published authors.

Submit your manuscript here: https://www.dovepress.com/international-journal-of-general-medicine-journal 